\title{
The Quasi-Longitudinal Approximation in the Generalized Theory of Radio Wave Absorption ${ }^{1}$
}

\author{
Robert F. Benson ${ }^{2}$ \\ Contribution from the Geophysical Institute, University of Alaska, College, Alaska
}

(Received August 5, 1963)

\begin{abstract}
Sample calculations for the case of nondeviative absorption indicate that the generalized magneto-ionic expression for the absorption coefficient for longitudinal radio wave propagation can be used with great accuracy even when the direction of propagation deviates greatly from longitudinal if the gyrofrequency $s$ in the equation is replaced by $\omega_{L}=|s \cos \phi|$, where $\phi$ is the angle between the propagation vector and the earth's lines of magnetic force. Curves indicating the percent error introduced by using the modified longitudinal equation (i.e. using $\omega_{L}$ ), in place of using the more involved expression for arbitrary $\phi$, are presented for the ordinary and extraordinary components for values of $\phi$ and $f$ between 10 to $50^{\circ}$ and 1 to 50 $\mathrm{Mc} / \mathrm{s}$, respectively. In the case of the extraordinary component, for frequencies in the 1 to $4 \mathrm{Mc} / \mathrm{s}$ range, greater accuracy is obtained by using the unmodified longitudinal equation even when $\phi \neq 0$ rather than introducing $\omega_{L}$. For both components the percent error introduced by using the simplified longitudinal expression, modified by using $\omega_{L}$ in place of $s$, is less than 1 percent for all values of $\phi$ up to $50^{\circ}$ when the frequency is $15 \mathrm{Mc} / \mathrm{s}$ or higher.
\end{abstract}

\section{Introduction}

The attenuation of an electromagnetic wave as it passes through an ionized medium can be described by the following expression:

$$
E=E_{0} e^{-\int^{s_{k} d s}}
$$

where

$E=$ amplitude of the electric field of the wave after having traveled a distance $s$ in the medium

$E_{0}=$ amplitude of the electric field of the incident wave

$\kappa=$ absorption coefficient.

The absorption coefficient $\kappa$ is given by

$$
\kappa=\frac{\omega \chi}{c}
$$

where $\omega$ is the angular frequency of the radio wave, $c$ is the velocity of light in vacuum, and $\chi$ is the imaginary part of the complex refractive index $n$ which is written as

$$
n=\mu-i \chi
$$

When the wave propagates parallel to the direction of the earth's magnetic field (longitudinal propaga-

\footnotetext{
1 This research was supported by the Air Force Cambridge Research Laboratories, Office of Aerospace Research under Contract No. A F 19(604)-8833.

tories, Office of Aerospace Research under Contract No. AF 19(604)-8833.
2 Present address, Department of Astronomy, University of Minnesota, Min${ }^{2}$ Present addres.
neapolis, Minn.
}

$709-774-64-6$ tion) the classical, or Appleton-Hartree, magnetoionic theory gives

$$
\kappa=\frac{1}{2 c \mu} \frac{\omega_{0}^{2} \nu_{A H}}{(\omega \pm s)^{2}+\nu_{A H}^{2}}=\frac{\left(5.31 \times 10^{-6}\right)}{\mu} \frac{N \nu_{A H}}{(\omega \pm s)^{2}+\nu_{A H}^{2}}
$$

where

$\omega_{0}^{2}=\frac{N e^{2}}{\epsilon_{0} m}\left(\operatorname{radians}^{2} / \mathrm{sec}^{2}\right)$

$N=$ electron density $\left(\mathrm{m}^{-3}\right)$

$e=$ electron charge (coulomb)

$m=$ electron mass $(\mathrm{kgm})$

$\epsilon_{0}=$ capacitivity (or permittivity) of free space (farads $/ \mathrm{m}$ )

$\mu=$ real component of the complex refractive index

$\omega=$ angular radiofrequency (radians/sec)

$s=$ angular gyrofrequency of the electrons due to the earth's total magnetic field in the region of interest (radians/sec). The plus sign is used for the ordinary wave and the minus sign for the extraordinary wave.

$\nu_{A H}=$ an average value for the electron collision frequency $\left(\mathrm{sec}^{-1}\right)$. The subscript $A H$ is used to distinguish between this average value and the average value used in the generalized magneto-ionic theory .

(Rationalized mks units are used throughout this paper.)

If the propagation vector makes an angle $\phi$ with respect to the direction of the earth's magnetic field the above equation can still be used, under certain conditions, if $s$ is replaced by $\omega_{L}=|s \cos \phi|$. The 
equation is then commonly referred to as the quasilongitudinal approximation [Ratcliffe, 1959, section 13.6; Ratcliffe uses unrationalized mks units].

A similar approximation has not been provided in the case of the generalized magneto-ionic theory which considers the effect of the velocity distribution function for the electrons. Sen and Wyller [1960] give generalized expressions for the complex refractive index for arbitrary $\phi$, for $\phi=0$, and for $\phi=\pi / 2$. The condition $\phi=0$ will be considered in the present discussion. In this case, the expression for $\kappa$ is found by combining eqs (39), (41), and (43) in the paper by Sen and Wyller [1960] to obtain

$$
\begin{aligned}
\kappa=\frac{1}{2 c \mu} \frac{\omega_{0}^{2}}{\nu_{m}}\left\{\frac{5}{2} \bigodot_{5 / 2}\left(\frac{\omega \pm s}{\nu_{m}}\right)\right\} \\
=\frac{\left(5.31 \times 10^{-6}\right)}{\mu} \frac{N}{\nu_{m}}\left\{\frac{5}{2} C_{5 / 2}\left(\frac{\omega \pm s}{\nu_{m}}\right)\right\}
\end{aligned}
$$

where $\nu_{m}$ is the mean electron collision frequency associated with the most probable electron speed. (In (4) it is not specified which average velocity, i.e., $v_{m}, \bar{v}$, or $v_{\text {rms }}$, the term $\nu_{A H}$ is to be associated with.) The $C_{5 / 2}$ function in (5) has been tabulated by Dingle, Arndt, and Roy [1957] for parameter values ranging from 0 to 20 . The other symbols have the same meaning as in (4). Note: the sign convention with $s$ is the same as in (4) but is opposite to that of Sen and Wyller [1960]; with this choice of signs, the same as was used by Chorbajian, Sugiura, and Parthasarathy [1962], the extraordinary wave is absorbed more strongly than the ordinary wave.

In the ionospheric literature, one commonly encounters the application of the classical quasi-longitudinal approximation to the equations of the generalized magneto-ionic theory without qualifying comments. In so doing, the formal simplicity of the longitudinal eq (5), modified by using $\omega_{L}=|s \cos \phi|$ in place of $s$, is retained (for $\phi \neq 0$ ) rather than using: the more involved expression for arbitrary $\phi$. The purpose of this paper is to determine under what conditions this procedure is justified. The investigation is based on sample calculations using both the most general expression and the longitudinal expression for $\kappa$ (for various values of $\omega$ and $\phi$ ) and calculating the percent error introduced by using the longitudinal expression when $\phi \neq 0$.

\section{Discussion}

Chorbajian, Sugiura, and Parthasarathy [1962] have calculated the absorption in decibels per kilometer path (designated by the parameter $A$ ), for radio waves of frequencies 1 to $50 \mathrm{Mc} / \mathrm{s}$ in the height range of approximately 20 through $100 \mathrm{~km}$. The generalized expression for the absorption coefficient $\kappa$, as derived from Sen and Wyller [1960], was used to calculate $A$ for values of $\phi$ from zero to $80^{\circ}$. Their results made it extremely easy to compare the simplified expression for longitudinal propagation $(\phi=0)$, as given by (5), with the complete expression for arbitrary direction of propagation for various values of $\phi$.
The parameter $A$ is defined as follows:

$$
\begin{aligned}
A & =-20 \log _{10}\left(E / E_{0}\right)=-20 \log _{10} e^{-\mathcal{S}_{\kappa} d h} \\
& =-20 \log _{10} e^{-\kappa\left(10^{3} \mathrm{~m}\right)} \\
& =\left(20 \times 10^{3}\right) \kappa \log _{10} e \mathrm{db} / \mathrm{km} .
\end{aligned}
$$

When the general expression for $\kappa$ is used, i.e., for arbitrary angle of propagation, the parameter $A$ will be designated as $A_{G}$. When the longitudinal expression for $\kappa$ is used, i.e., eq (5), the parameter $A$ will be designated as $A_{L}$. Combining (5) and (6) gives the following expression for nondeviative absorption $(\mu=1)$ :

$$
A_{L}=\frac{1.15 \times 10^{5}}{\nu_{m}} C_{5 / 2}\left(\frac{\omega \pm s}{\nu_{m}}\right)
$$

when $N=10^{6} \mathrm{~m}^{-3}$. $\quad N$ is set equal to $10^{6} \mathrm{~m}^{-3}$ so that $A_{L}$ from (7) can be compared with $A_{G}$ as given by Chorbajian, Sugiura, and Parthasarathy [1962] who also used this value. (Note: the above authors used cgs units.) The quantity

$$
\frac{A_{G}-A_{L}}{A_{G}} \times 100
$$

was calculated for $\phi$ ranging from zero to $50^{\circ}$ and $f$ $=\omega / 2 \pi$ ranging from 1 to $50 \mathrm{Mc} / \mathrm{s}$.

The $C_{5 / 2}$ function in (7) was evaluated using the method of Chorbajian, Sugiura, and Parthasarathy [1962], i.e., using the tabulated values of Dingle, Arndt, and Roy [1957] and interpolating from Newton's forward difference formula (to second order differences) for parameter values between 0 and 0.2 and using Bessel's central difference formula (to third order differences) for parameter values between 0.2 and 19.0. For parameter values greater than 19.0 the integral was approximated as $1 / x^{2}$.

The percent error, as given by (8), was calculated for the ordinary and extraordinary components with $\omega_{L}$ in place of $s$, i.e. where $\left(\omega+\omega_{L}\right) / \nu_{m}$ and $\left|\omega-\omega_{L}\right| / \nu_{m}$, respectively, were used in (7). (The absolute value was used because $C_{5 / 2}(x)$ is an even function of $x$ ). The same calculations were performed before introducing $\omega_{L}$ into the longitudinal equation in order to illustrate the $\phi$ and $f$ dependence of (8) when the unmodified form of $A_{L}$ is used. The results are presented in figures 1,2 , and 3 corresponding to three different values for $\nu_{m}$. The absolute value of (8) is represented in the figures since $A_{G}-A_{L}(s)>0$ and $A_{G}-A_{L}\left(\omega_{L}\right)>0$ for $f$ between 1 and $50 \mathrm{Mc} / \mathrm{s}$ for the ordinary component, whereas for the extraordinary component $A_{G}-A_{L}(s)<0$ for $f$ between 1 and 50 $\mathrm{Mc} / \mathrm{s}, A_{G}-A_{L}\left(\omega_{L}\right)>0$ for $f$ greater than approximately $1.5 \mathrm{Mc} / \mathrm{s}\left(\omega-\omega_{L}>0\right)$, and $A_{G}-A_{L}\left(\omega_{L}\right)<0$ for $f$ less than the above value. The main points to observe on these figures are the following:

Ordinary Component. The solid curves indicate the percent error introduced by using the modified longitudinal equation, i.e., using $\omega_{L}$ in place of $s$, 

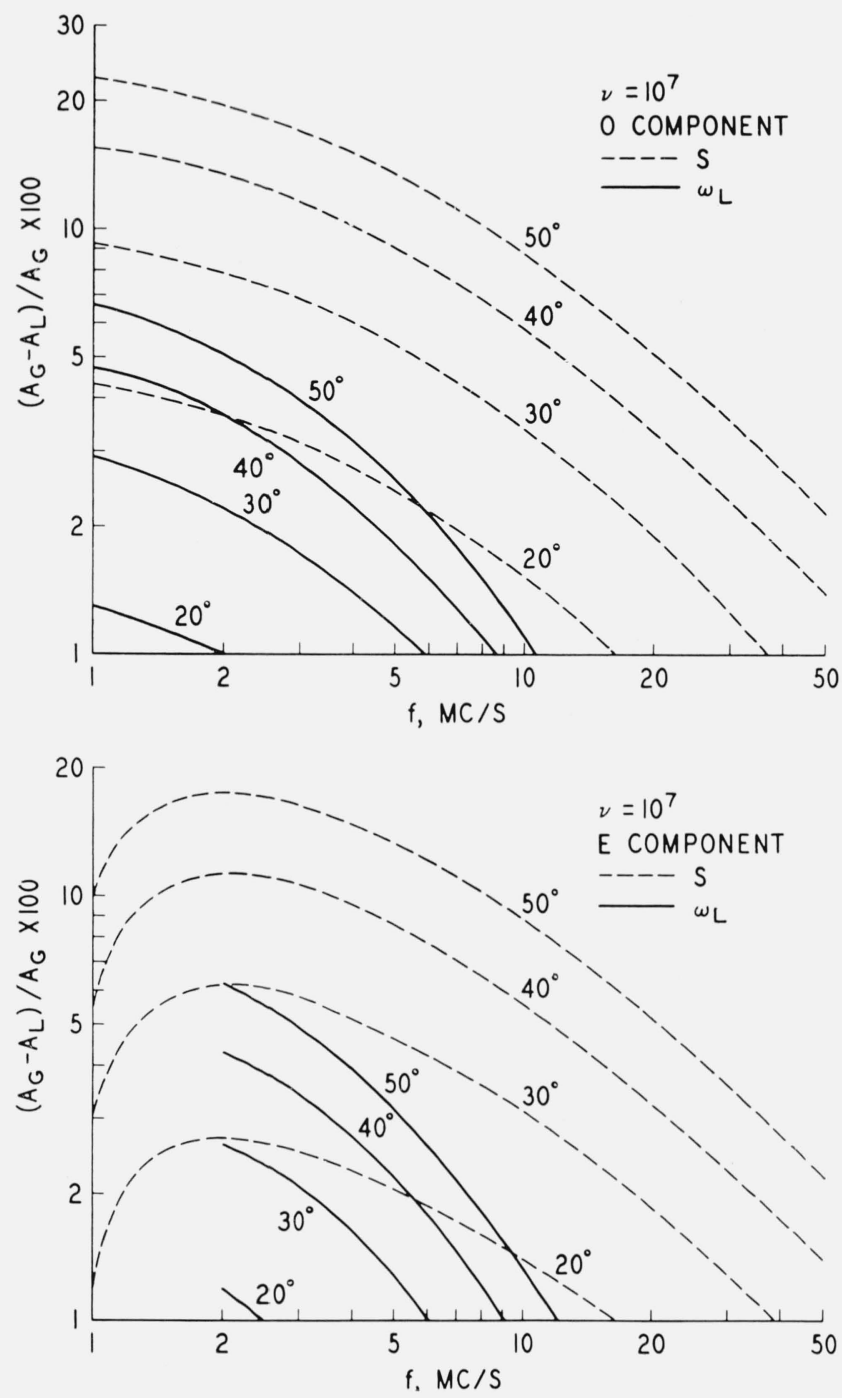

Figure 1. Percent error in the generalized magneto-ionic theory QL approximation when $v=10 .^{7}$

Top: Ordinary component [0]. AG represents the absorption per kilometer path per electron as calculated from the generalized magneto-ionic theory expression for arbitrary value of $\phi ; A_{L}$ represents the same quantity as calculated from sion for arbitrary value of $\emptyset ; A_{L}$ represents the same quantity as calculated from The dashed curves, for various values of $\phi$, are obtained when the unmodified longitudinal expression is used to calculated $A_{L}$; the solid curves are obtained longitudinal expression is used to calculated $A_{L}$; the solid curves are obtained
when the modified longitudinal expression, i.e., $s$ is replaced by $\omega_{L}=|s \cos \phi|$, is used to calculate $A_{L}$. Bottom: Extraordinary component [E]. Same caption as for the ordinary component.

when $\phi \neq 0$. For $f \geq 15 \mathrm{Mc} / \mathrm{s}$ the error is less than 1 percent for all values of $\phi$ up to $50^{\circ}$.

\section{Extraordinary Component.}

1. For frequencies less than a certain critical value, between 1 and $4 \mathrm{Mc} / \mathrm{s}$ depending on the value of $\nu_{m}$ and $\phi$, greater accuracy is obtained using the unmodified longitudinal equation even when $\phi \neq 0$ rather than introducing $\omega_{L}$. Thus in this frequency range one must resort to the complete expression for arbitrary $\phi$ if accuracy greater than that indicated by the dotted curves is desired.

2 . The solid curves were terminated at $f=2 \mathrm{Mc} / \mathrm{s}$ because, as was mentioned earlier, the term $A_{G}-$
$A_{L}\left(\omega_{L}\right)$ changes sign between $f=2 \mathrm{Mc} / \mathrm{s}$ and $f=1$ $\mathrm{Mc} / \mathrm{s}$. In this frequency range the percent error increases very rapidly and the curve could not be extended on the basis of one additional point $(f=1 \mathrm{Mc} / \mathrm{s})$.

3. For frequencies of $15 \mathrm{Mc} / \mathrm{s}$ and higher, the error is less than 1 percent for all values of $\phi$ up to $50^{\circ}$ when $\omega_{L}$ is used in place of $s$.

Only three values of $\nu_{m}$ were considered because as $\nu_{m}$ increases the percent error decreases and as $\nu_{m}$ decreases the dominant absorption, for the lower frequencies, is deviative absorption $(\mu \neq 1)$ rather than nondeviative absorption $(\mu=1)$.

It should be pointed out that Chorbajian, Sugiura, and Parthasarathy [1962] arbitrarily used Nicolet's (1959) collision frequency profile for $\nu_{m}$. Their values of $h, \nu_{m}$, and $s$ appropriate to figures 1,2 , and 3 are the following:

\begin{tabular}{|c|c|c|c|}
\hline Figure & $h(\mathrm{~km})$ & $\nu_{m}\left(\sec ^{-1}\right)$ & $s\left(\right.$ radians sec $\left.{ }^{-1}\right)$ \\
\hline 1 & 70.5 & $10^{7}$ & $9.6679237 \times 10^{6}$ \\
\hline 2 & 84.5 & $10^{6}$ & 9. $6050611 \times 10^{6}$ \\
\hline 3 & 99.0 & $10^{5}$ & $9.5405268 \times 10^{6}$ \\
\hline
\end{tabular}

If the above heights are changed so as to agree with the $\nu_{m}$ profile of Kane [1960 and 1961] which is preferable [Parthasarathy, 1963], the corresponding $s$ values will change slightly $(\approx 0.1 \%)$; the percent error values given in the above figures will change even less.

\section{Summary}

The longitudinal expression for the absorption coefficient in the generalized theory (5) is sufficient for most applications over a wide range of frequencies and over a wide range of $\phi$ angles, when $s$ is replaced by $\omega_{L}=|s \cos \phi|$. For frequencies in the 1 to $4 \mathrm{Mc} / \mathrm{s}$ range, however, the introduction of $\omega_{L}$ in the longitudinal equation for the extraordinary component either has very little effect or produces greater errors than result from using the unmodified longitudinal equation when $\phi \neq 0$. In this frequency range, it may be necessary to use the complete expression for arbitrary $\phi$.

I am thankful to R. Parthasarathy for encouraging this investigation and for many helpful discussions. I also thank S. Young for programming the University of Alaska IBM 1620 Computer.

\section{References}

Chorbajian, J., M. Sugiura, and R. Parthasarathy, (Nov. 1962), Radio wave absorption coefficients based on Sen-Wyller magneto-ionic formula, Tech. Report, Univ. of Alaska, Geophys. Inst. Rept. 132.

Dingle, R. B., D. Arndt, and S. K. Roy, (1957), The integrals

$$
\begin{aligned}
\bigodot_{p}(x) & =(p !)^{-1} \int_{0}^{\infty} \epsilon^{p}\left(\epsilon^{2}+x^{2}\right)^{-1} e^{-\epsilon} d \epsilon \text { and } D_{p}(x) \\
& =(p !)^{-1} \int_{0}^{\infty} \epsilon^{p}\left(\epsilon^{2}+x^{2}\right)^{-2} e^{-\epsilon} d \epsilon
\end{aligned}
$$



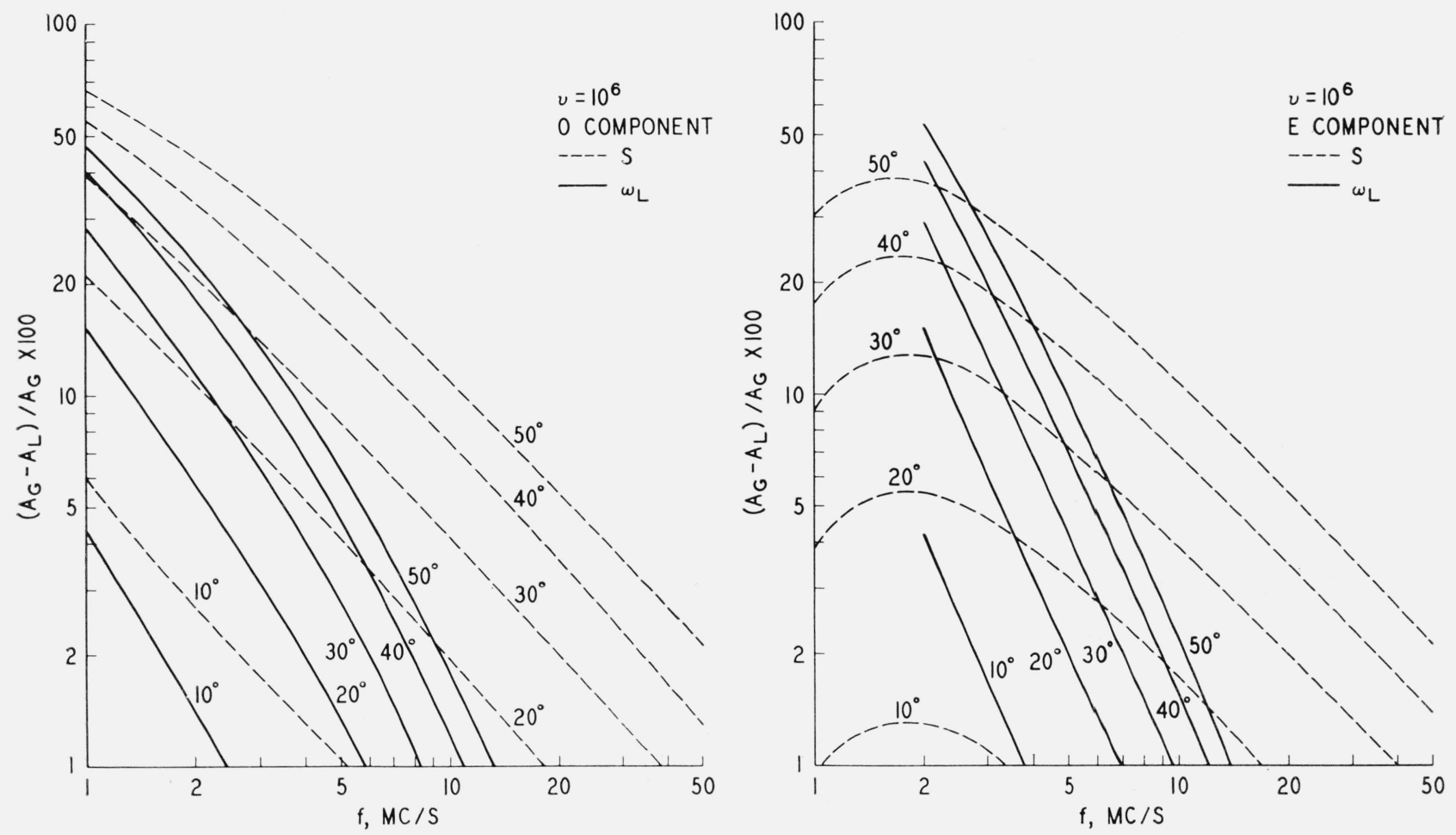

Figure 2. Percent error in the generalized magneto-ionic theory $Q L$ approximation when $v=10 .{ }^{6}$ Left: Ordinary component [0]. Right: Extraordinary component [E]. See caption with figure 1.
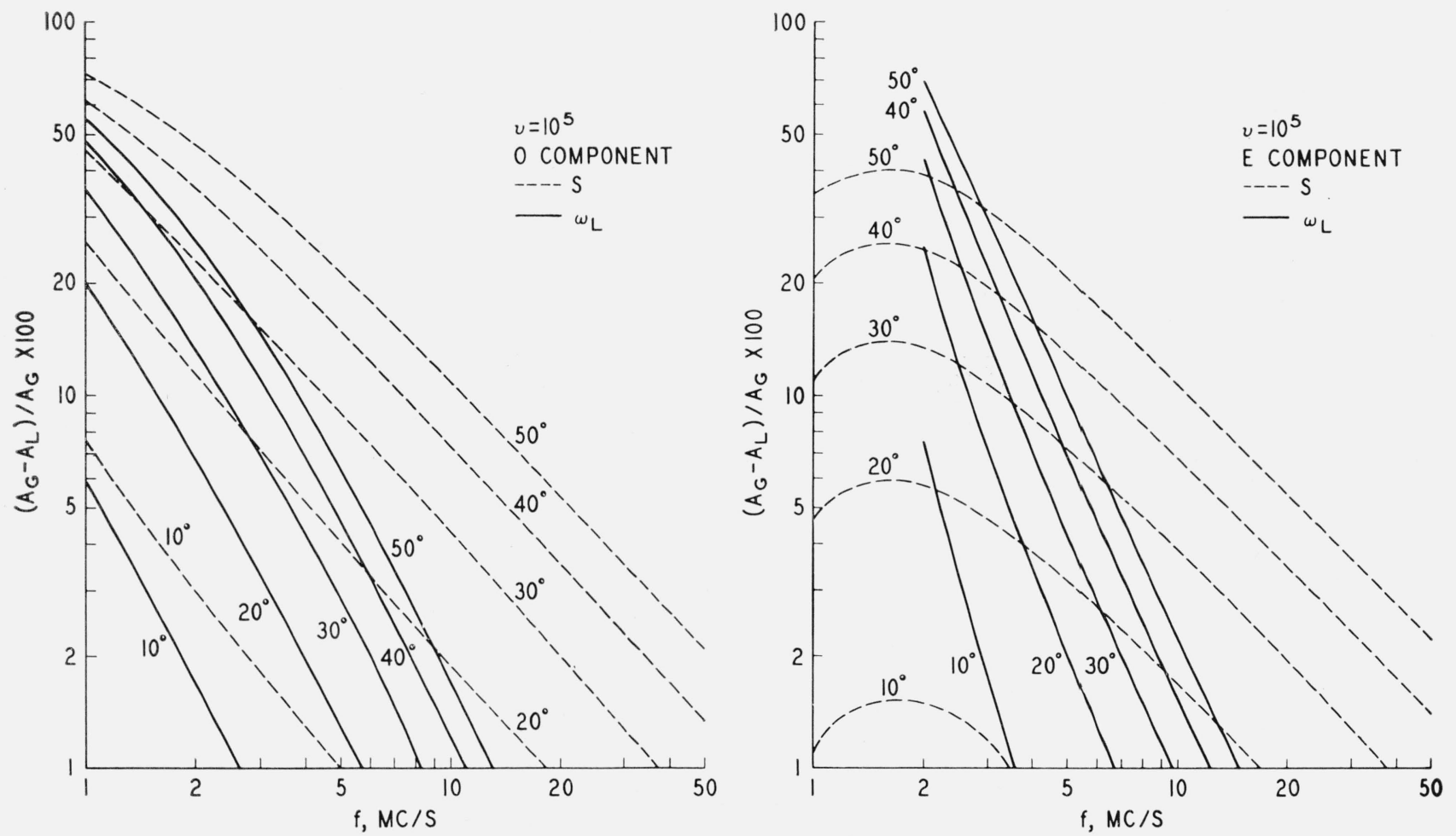

Figure 3. Percent error in the generalized magneto-ionic theory $Q L$ approximation when $v=10.5$ Left: Ordinary component [0]. Right: Extraordinary component [E]. See caption with figure 1. 
and their tabulation, Appl. Sci. Res. 6B, 155-164, (The Hague, Netherland).

Kane, J. A., (Nov. 1960), Reevaluation of ionosphere electron densities and collision frequencies derived from rocket measurements of refractive index and attenuation, NASA Tech. Note D-503.

Kane, J. A., (1961), Reevaluation of ionosphere electron densities and collision frequencies derived from rocket measurements of refractive index and attenuation, J. Atmospheric Terrest. Phys. 23, 338-347.
Nicolet, M., (1959), Collision frequency of electrons in the terrestrial atmosphere, Phys. Fluids $\mathbf{2}, 95-99$.

Parthasarathy, R., (1963), (private communications).

Ratcliffe, J. A., (1959), The magneto-ionic theory and its applications to the ionosphere, (Cambridge Univ. Press, London).

Sen, H. K., and A. A. Wyller, (1960), On the generalization of the Appleton-Hartree magneto-ionic formulas, J. Geophys. Res. 65, 3931-3950.

(Paper 68D2-336) 